\title{
Nifedipine Induced Gingival Hyperthropy in a Patient with Systemic Sclerosis Associated Raynaud's Phenomenon
}

The Editor

Sir,

Systemic sclerosis ( $\mathrm{SSc}$ ) is a connective tissue disease in which autoimmunity, vasculopathy, and fibrosis may play crucial roles in the SSc aetiopathogenesis (1). Raynaud's phenomenon (RP) is the major vascular involvement in SSc. Raynaud's phenomenon is a vasospastic change in the vascular system. It may be primary RP (due to cold, emotional stress, etc) or secondary RP (primarily underlying connective tissue disease such as $\mathrm{SSc}$ ). Calcium channel blockers have been used for the treatment of RP (2). It has been suggested that cyclosporine, antiepileptic drugs (ie phenytoin) and nifedipine may cause gingival overgrowth (3).

A 55-year-old woman was admitted to our out-patient clinic for complaints of dry mouth, decreased mouth opening, gingival enlargement (Figure).
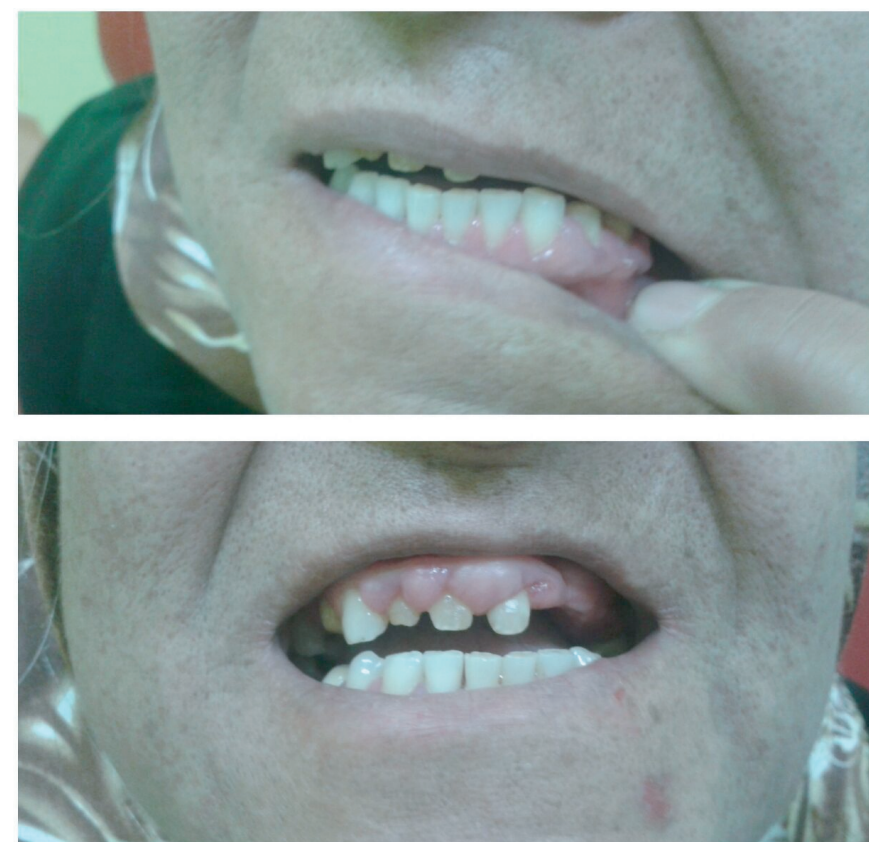

Figure: Gingival hypertrophy and reduced mouth opening of the patient.

She had been diagnosed as SSc in 2008. She had antinuclear antibody (ANA) and anti-scl-70 (topoisomerase) positivity then. She was administered nifedipine, aspirin for RP and methyprednisolone, cyclophosphamide for interstitial lung involvement and hydroxychloro-quine, colchicine, non-steroidal anti-inflammatory drugs for musculoskelatal involvement. On her examination, we detected restricted mouth opening, gingival hypertrophy (Figure), sclerodactyly, cold-pale hands, digital pitting and ulcer scars, flexion contractures, telengiectasias, restricted pulmoner capacity and coarse crackles (rales). She had been using nifedipine for a long-time and she had no other drug history that could cause gingival hypertrophy. Hence, we stopped the use of nifedipine and started losartan potassium $50 \mathrm{mg} /$ day.

On the course of SSc, periodontal and other oral findings may be seen. Nifedipine-induced gingival hypertrophy was described by Lederman, firstly (4). As we know, fibroblasts synthesize exracellular collagen and play the major roles in SSc pathogenesis. Nifedipine has potential effects on the gingival fibroblast (5). Gingival fibroblasts overproduce collagen and other extracellular matrix compounds when nifedipine interacts with them (5). The production and secretion of proteinases especially collagenases are reduced by the effect of nifedipine on gingival fibroblasts (5). Impaired oral hygiene, inflammation, existing inflammatory diseases (ie $\mathrm{SSc}$ ) or conditions, inflammatory cytokines (ie İnterleukin-1 $\beta$, TGF- $\beta$ ) may cause nifedipine and gingival fibroblasts interactions so gingival hypertrophy develops (5). Angiotensin II receptor blockers (ie losar$\tan$ ) can be used instead of calcium channel blockers in the treatment of RP if gingival hypertrophy is observed (5). In addition, it has been declared that use of losartan potassium $25 \mathrm{mg}$ /day for two months instead of nifedipine could reduce the gingival enlargement (5). The differential diagnosis of gingival overgrowth consists of impaired oral hygiene, autoimmune-inflammatory diseases (ie SSc), chronic ginigivitis, hyperplastic gingivitis, hereditary gingival fibromatosis, pregnancy associated gingivitis, sarcoidosis, Chron's disease, leukaemia, amyloidosis, granulomatosis with polyangitis (Wegener), lysosomal and collagen storage disorders (mucolipidosis, mucosaccaridosis), thyroid dysfunctions (hypothyroidism, Hashimoto thyroiditis) and vitamin deficiencies (ie vitamin $\mathrm{C}$ ). 
Although SSc is an unsolved puzzle in medical practice, the clinicians should be aware of which drug has potential side effects to her/his patients. In particular, commonly precribed drugs such as calcium channel blockers for the treatment of RP in SSc must be used with caution.

Keywords: Gingival hypertrophy, nifedipine, raynaud phenomenon

A Şahin 1 , A İhan ${ }^{2}$

From: ${ }^{1}$ Department of Rheumatology-internal medicine and ${ }^{2}$ Department of internal medicine, Cumhuriyet University School of medicine, Sivas, Turkey.
Correspondence: $\operatorname{Dr}$ A Sahin, Department of Rheumatology-internal medicine, Cumhuriyet University School of medicine, 58140, Sivas / Turkey.

Email:dralsahin@hotmail.com

\section{REFERENCES}

1. Hudson M, Fritzler MJ. Diagnostic criteria of systemic sclerosis. J Autoimmun 2014 Feb-Mar;48-49:38-41.doi:10.16/j.jaut.2013.11.004. Epub 2014 Jan 22.

2. Sinnathurai P, Schrieber L. Treatment of Raynaud phenomenon in systemic sclerosis. Intern Med J 2013; 43: 476-83.

3. Nakib N, Ashrafi SS. Drug-induced gingival overgrowth. Dis Mon 2011; 57: $225-30$

4. Lederman D, Lumerman H, Reuben S, Freedman PD. Gingival hyperplasia associated with nifedipine therapy. Oral Surg 1984; 57: 620-22.

5. Sam G, Sebastian C. Nonsurgical management of nifedipine induced gingival overgrowth. Case Rep Dent 2014; 2014: 741402. 